\title{
Through-Focal HAADF-STEM Analysis of Dislocation Cores in a High-Entropy Alloy
}

\author{
T.M. Smith ${ }^{1}$, B.D. Esser ${ }^{1}$, M.S. Hooshmand ${ }^{1}$, E.P. George ${ }^{2}$, F. Otto ${ }^{2}$, M. Ghazisaeidi ${ }^{1}$, D.W. McComb ${ }^{1}$, \\ M.J. Mills ${ }^{1}$ \\ 1. Center for Electron Microscopy and Analysis, The Ohio State University, Columbus, OH \\ 2. Institute for Materials, Ruhr University Bochum, Bochum, Germany
}

High-entropy alloys (HEAs) are a new class of multi-component alloys that exhibit surprising characteristics, [1] including very large strain hardening rates, large fracture toughness at room temperature [2], and a strong temperature dependence of yield strength at or below room temperature. These properties are closely linked to nano-twinning and dislocation-mediated plasticity, yet little experimental work has explored dislocation dissociation, stacking fault energy, or core structures in these alloys [3]. In this study, an HEA, containing 5 elements $(\mathrm{Cr}, \mathrm{Co}, \mathrm{Mn}, \mathrm{Fe}$, and $\mathrm{Ni}$ ) with equiatomic composition was deformed to a 5\% plastic strain at room temperature [4]. Post-mortem 3mm disks were electro-polished using a solution consisting of $21 \%$ Perchloric acid and $79 \%$ Acetic acid and analyzed using a probe-corrected $\operatorname{Titan}^{3} 80-300 \mathrm{kV}$ along a [110] zone axis. Highly planar deformation was first observed by Otto et al. [5] and was active for this study as well. This planar deformation, involving dislocation arrays on $\{111\}$ slip systems, may imply the existence of short-range order, low stacking fault energy (SFE), and/or supplementary displacements in the wake of dislocations.

Smith et al. [6] previously demonstrated that high and medium angle annular dark field scanning transmission electron microscopy (HAADF/MAADF-STEM) could effectively be used to determine the misalignment of a dislocation through foil thickness. This misalignment created a contrast "plume" when imaged in a MAADF condition. Recently, Smith et al. revealed the presence of a broad distribution of stacking fault widths, suggesting the concept of a "local" stacking fault energy in HEAs which affect the the dislocation dissociation and may play a role in how these dislocations glide [7]. To further explore this misalignment and how it relates to the dislocation core structure, through-focal HAADF-STEM imaging was employed. Acquisition of a through-focal STEM series was shown to enable detection of the crystal rotation in association with the "Eshelby twist" around screw dislocations [8]. This technique has been employed presently to create a 3D analysis of dislocation cores in the Cantor alloy as shown in Figure 1(a) and 1(b). Changing defocus allowed different depths along a dislocation line to be imaged, allowing for a three dimensional analysis of the whole dislocation core. The field of focus (z) was calculated using [9]:

$$
z=\frac{\lambda}{\alpha^{2}}
$$

where $\lambda$ was the electron wavelength (which at $300 \mathrm{kV}$ was $.00224 \mathrm{~nm}$ ) and $\alpha$ was the convergence angle $(22 \mathrm{mrad})$. Therefore, the depth of field for this study was $4.6 \mathrm{~nm}$. A nano-hole was drilled through the sample near the dislocation to act as a marker and reveal changes in the dislocation's location and dissociation distance. Two different dislocation types were analyzed using this technique. One with a short contrast "plume" attached to it and another with a much longer one - the latter shown in Figure 2. A gray box with a red outline is placed over the dislocations stacking fault and represents the dislocation's location and dissociation width. A series of different defocal images were taken for both types of dislocations and aligned using ImageJ [10]. For both dislocations, the dissociation distance changed along the dislocation line; however, the dislocation with a long plume showed a much larger variation in stacking fault width as shown in Figure 2(b), 2(c), and 2(d). These findings demonstrate the 
unique capability of through-focal HAADF imaging for probing dislocation structure information in 3D at atomic-scale. These results will be discussed in the context of the concept of a "local" SFE in this HEA, and in relationship to the unique macro-behavior exhibited by these alloys.

References:

[1] J.-W. Yeh et al. Adv. Eng. Mater. 6 (2004) 299-303.

[2] B. Gludovatz et al. Science. 345 (2014) 1153-1158.

[3] Z. Zhang et al. Nat. Commun. 6 (2015) 10143.

[4] B. Cantor et al. Mater. Sci. Eng. A. 375-377 (2004) 213-218.

[5] F. Otto et al. Acta Mater. 61 (2013) 5743-5755.

[6] T.M. Smith et al. Microsc. Microanal. 21 (2015) 2205-2206.

[7] T.M. Smith et al. Acta Mater. Pending Submission (2016).

[8] J.E. Allen et al. Nat. Nanotechnol. 3 (2008) 168-173.

[9] P.D. Nellist et al. Microsc. Microanal. 14 (2008) 82-88.

[10] C.A. Schneider et al. Nat. Methods. 9 (2012) 671-675.

[11] Support for this program has been provided by the National Science Foundation under DMR1508505. BDE and DWM acknowledge the Center for Emergent Materials: an NSF MRSEC under award number DMR-1420451, as well as partial support by an allocation of computing time from the Ohio Supercomputer Center.

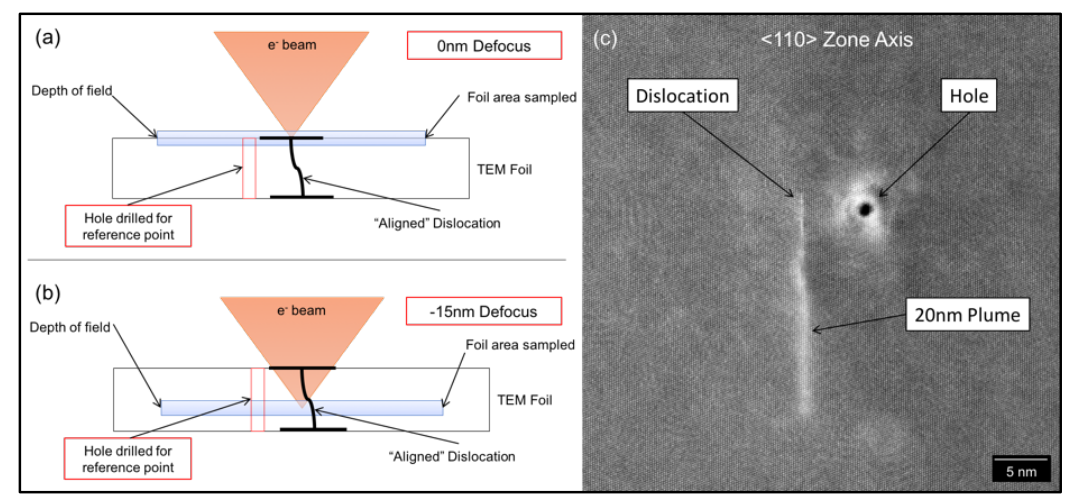

Figure 1: A schematic of the through-focal STEM technique at (a) 0nm defocus and (b) -15nm defocus. (c) A MAADF-STEM image showing an example of the setup for the through-focal STEM.
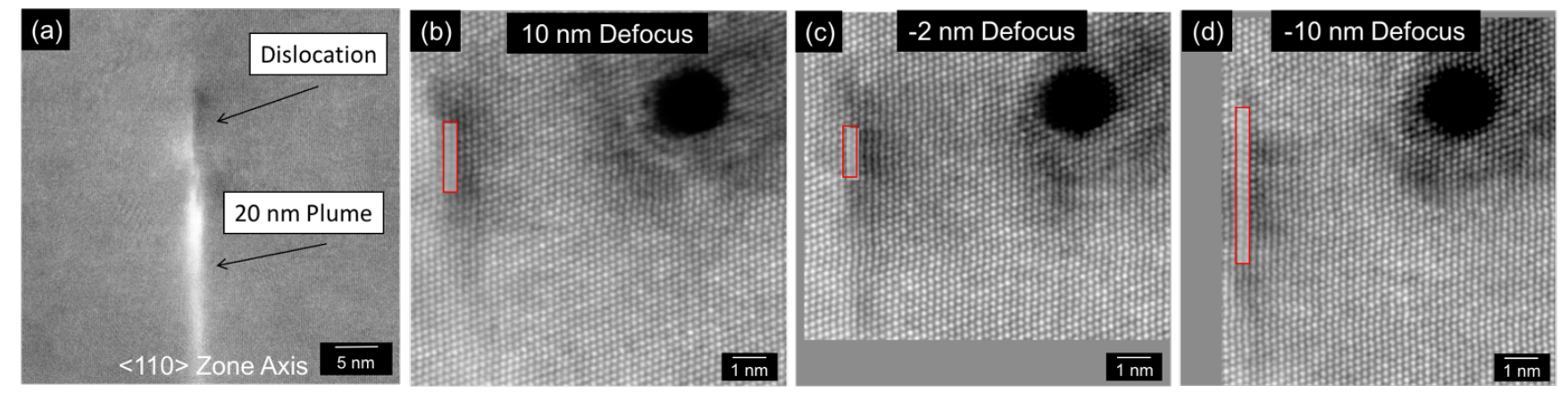

Figure 2: (a) A dislocation with a long (20nm) contrast "plume" attached to it that was imaged edge-on at different defocus values (b) 10nm defocus (c) $-2 \mathrm{~nm}$ defocus and (d) $-10 \mathrm{~nm}$ defocus. 